\title{
The Existence of Affine Structures on the Borel Subalgebra of Dimension 6
}

\author{
Edi Kurniadi ${ }^{1 *}$, Ema Carnia², and Herlina Napitupulu ${ }^{3}$ \\ ${ }^{1-3}$ Department of Mathematics, Faculty of Mathematics and Natural Sciences, Universitas Padjadjaran \\ Jln. Raya Bandung Sumedang KM 21 Jatinangor, Jawa Barat 45363, Indonesia \\ 1edi.kurniadi@unpad.ac.id; ${ }^{2}$ ema.carnia@unpad.ac.id; ${ }^{3}$ herlina@unpad.ac.id
}

Received: $20^{\text {th }}$ July 2020/ Revised: $21^{\text {st }}$ Desember 2020/ Accepted: $6^{\text {th }}$ January 2021

\begin{abstract}
How to Cite: Kurniadi, E., Carnia, E., \& Napitupulu, H. (2021). The Existence of Affine Structures on the Borel Subalgebra of Dimension 6. ComTech: Computer, Mathematics and Engineering Applications, 12(1), 45-52. https://doi.org/10.21512/comtech.v12i1.6581
\end{abstract}

\begin{abstract}
The notion of affine structures arises in many fields of mathematics, including convex homogeneous cones, vertex algebras, and affine manifolds. On the other hand, it is well known that Frobenius Lie algebras correspond to the research of homogeneous domains. Moreover, there are 16 isomorphism classes of 6-dimensional Frobenius Lie algebras over an algebraically closed field. The research studied the affine structures for the 6-dimensional Borel subalgebra of a simple Lie algebra. The Borel subalgebra was isomorphic to the first class of Csikós and Verhóczki's classification of the Frobenius Lie algebras of dimension 6 over an algebraically closed field. The main purpose was to prove that the Borel subalgebra of dimension 6 was equipped with incomplete affine structures. To achieve the purpose, the axiomatic method was considered by studying some important notions corresponding to affine structures and their completeness, Borel subalgebras, and Frobenius Lie algebras. A chosen Frobenius functional of the Borel subalgebra helped to determine the affine structure formulas well. The result shows that the Borel subalgebra of dimension 6 has affine structures which are not complete. Furthermore, the research also gives explicit formulas of affine structures. For future research, another isomorphism class of 6-dimensional Frobenius Lie algebra still needs to be investigated whether it has complete affine structures or not.
\end{abstract}

Keywords: Affine structures, Borel subalgebras, Frobenius Lie algebras

\section{INTRODUCTION}

There are 16 isomorphism classes of 6-dimensional Frobenius Lie algebras over an algebraically closed field (Csikós \& Verhóczki, 2007). One of them is a Frobenius Lie algebra whose basis is
$H:=\left\{x_{i}\right\}_{i=1}^{6}$, and it is nothing but the first class of Csikós and Verhóczki's classification. The non-zero brackets of this Frobenius Lie algebra are given as follows:

$$
\begin{array}{ll}
{\left[x_{1}, x_{3}\right]=x_{3},} & {\left[x_{1}, x_{5}\right]=x_{5}} \\
{\left[x_{1}, x_{6}\right]=2 x_{6},} & {\left[x_{2}, x_{4}\right]=x_{4}} \\
{\left[x_{2}, x_{5}\right]=x_{5},} & {\left[x_{2}, x_{6}\right]=x_{6}} \\
{\left[x_{3}, x_{4}\right]=x_{5},} & {\left[x_{3}, x_{5}\right]=x_{6} .}
\end{array}
$$

In addition, this Frobenius Lie algebra is isomorphic to the Borel subalgebra of dimension 6 of the simple Lie algebra (Csikós \& Verhóczki, 2007). Therefore, the means of the Borel subalgebra in this research is nothing but the 6-dimensional Frobenius Lie algebra over the complex field $\mathbb{C}$. Indeed, the research also suggests that this Frobenius Lie algebra is maximal solvable.

The recent research of Frobenius Lie algebras has been done by Kurniadi and Ishi (2019). They obtained some results of 4-dimensional real Frobenius Lie algebras. Their main results stated that every irreducible unitary representation of Lie groups of 4-dimensional real Frobenius Lie algebras was squareintegrable. Moreover, they found the Duflo-Moore operators for these square-integrable representations which are denoted in the group of Fourier transforms.

On the other hand, the notions of principal elements of a Frobenius Lie algebra and their properties are studied by Diatta and Manga (2014). Furthermore, the discussion of a higher dimension of Frobenius Lie algebras is studied for the case of a semi-direct product of a vector space $V$ which is isomorphic to the real vector space $\mathbb{R}^{n}$ of dimension $n$ and a Lie subalgebra $h$ of the Lie algebra $g l(\mathrm{~V})$ of endomorphisms of $\mathrm{V}$. A matrix Lie group $g l(V)$ of the Lie algebra $\mathfrak{g l}(\mathrm{V})$ can be realized as invertible matrices of $n \times n$ dimension. The result of this formula has been done by Kurniadi (2019). In this case, it was proved the necessary and sufficient conditions for the direct sum $v \oplus h$ to be a 
real Frobenius Lie algebra. In another work, the notion of Frobenius solvable Lie algebras with abelian nilradical has been also investigated by Alvarez, Rodríguez-Vallarte, and Salgado (2018). On the other hand, the notion of left symmetric algebras is induced by symplectic structures. It can be observed that this induction can be applied to a Frobenius functional of a Frobenius Lie algebra. In other words, it is well known that if $\mathfrak{g}$ is a Frobenius Lie algebra, there is a certain linear functional, which is called Frobenius functional. The bilinear form on $\mathfrak{g}$ at that point is non-degenerate. The previous research introduces the notion of systems of commuting matrices associated with left-symmetric algebra structures (Diatta, Manga, \& Mbaye, 2020).

The research observes that this induction holds for a chosen Frobenius functional of the 6-dimensional Frobenius Lie algebra. Moreover, the research problem is to prove the existence of affine structures on Borel subalgebra of dimension 6 and show that this structure is not complete. The affine structures play important roles in many areas of mathematics, such as in convex homogeneous cones. The results can be improved to a higher dimension case. As the main result, the research tries to prove the following propositions.

Proposition 1. Let $\mathfrak{g}$ be a Lie subalgebra of a simple Lie algebra with basis $H:=\left\{x_{i}\right\}_{i=1}^{6}$ whose nonzero brackets are given by Equation (1), then $\mathfrak{g}$ is a Borel subalgebra. Moreover, $\mathfrak{g}$ is a Frobenius solvable Lie algebra, and it has affine structures written as following.

$$
\begin{aligned}
& x_{1}^{2}=-2 x_{1}, x_{1} x_{2}=-x_{1}, x_{1} x_{3}=-x_{3}, \\
& x_{1} x_{4}=0, x_{1} x_{5}=-x_{5}, x_{1} x_{6}=0, \\
& x_{2} x_{1}=-x_{1}, x_{2}^{2}=-x_{2}, x_{2} x_{3}=-x_{3}, \\
& x_{2} x_{4}=0, x_{2} x_{5}=0, x_{2} x_{6}=0, \\
& x_{3} x_{1}=-2 x_{3}, x_{3} x_{2}=-x_{3}, x_{3}^{2}=\frac{1}{2} x_{1}-x_{2}, \\
& x_{3} x_{4}=0, x_{3} x_{5}=-\frac{1}{2} x_{4}+\frac{1}{2} x_{6}, x_{3} x_{6}=0, \\
& x_{4} x_{1}=0, x_{4} x_{2}=-x_{4}, x_{4} x_{3}=-x_{5}, \\
& x_{4}^{2}=0, x_{4} x_{5}=0, x_{4} x_{6}=0, \\
& x_{5} x_{1}=-2 x_{5}, x_{5} x_{2}=-x_{5}, x_{5} x_{3}=-\frac{1}{2} x_{4}-\frac{1}{2} x_{6}, \\
& x_{5} x_{4}=0, x_{5}^{2}=-x_{4}, x_{5} x_{6}=0, \\
& x_{6} x_{1}=-2 x_{6}, x_{6} x_{2}=-x_{6}, x_{6} x_{3}=0, \\
& x_{6} x_{4}=0, x_{6} x_{5}=0, x_{6}^{2}=0 .
\end{aligned}
$$

The products on $\mathfrak{g}$ are defined in Equation (3) for positive integers of $1 \leq i, j \leq 6$. Furthermore, the affine structures on the Borel subalgebra $\mathfrak{g}$ written in Equation (2) are not complete.

$$
\mathrm{g} \times \mathrm{g} \exists\left(x_{i}, x_{j}\right) \mapsto x_{i} x_{j}:=x_{i} * x_{j} \in \mathrm{g} .
$$

The research will prove Proposition 1. Before proving the main result, it briefly reviews some basic notions of a Lie algebra, solvable Lie algebras, Frobenius Lie algebras, and affine structures. It starts with the notion of a Lie algebra as follows.
Definition 2 (Fujiwara \& Ludwig, 2015). Let $\mathfrak{g}$ be a vector space over a field of $\mathbb{F}=\mathbb{R}$ or $\mathbb{C}$. Let $\mathrm{g} \times \mathrm{g} \rightarrow \mathrm{g}$ be a bilinear map defined by $(x, y) \mapsto[x, y]$. If the following two conditions are satisfied $[x, x]=0$ and $[[x, y], z]=[x,[y, z]]+[y,[z, x]], \mathfrak{g}$ is said to be a Lie algebra.

Indeed, the basis and dimension of a Lie algebra can be understood from the notions of the basis and dimension of the vector space. Let $\mathfrak{g}$ and $g^{\prime}$ be Lie algebras over a field $\mathbb{F}=\mathbb{R}$ or $\mathbb{C}$. It recalls a notion of a homomorphism of the Lie algebra as a linear transformation of $\theta: \mathrm{g} \rightarrow \mathrm{g}^{\prime}$ of the vector spaces. It satisfies $\theta([x, y])=[\theta(x), \theta(y)]$ for all $x, y \in \mathrm{g}$. When the homomorphism of Lie algebra is bijective, it has a notion of an isomorphism of Lie algebra. Let $\mathfrak{g}$ be a Lie algebra whose the derived series on $\mathfrak{g}$ is defined as follows, for $k \in \mathbb{Z}_{>0}$.

$$
g^{(0)}=g, g^{(1)}=[g, g], \ldots g^{(k+1)}=\left[g^{(k)}, g^{(k)}\right] .
$$

Definition 3 (Hilgert \& Neeb, 2011). Let $\mathrm{g}$ be a Lie algebra. The Lie algebra $\mathfrak{g}$ is said to be solvable if there exists a positive integer of $k \in \mathbb{Z}_{>0}$, such as $\mathrm{g}^{(\mathrm{k})}=\{0\}$.

Let $G$ be a Lie group with a Lie algebra of $g$ and $\mathfrak{g}^{*}$ be the dual vector space of $\mathfrak{g}$. The Lie group $G$ acts on the Lie algebra $\mathfrak{g}$ by adjoint action, as denoted by $\mathrm{Ad}$, and $\mathrm{g}^{*}$ by coadjoint action as denoted by Ad*. The latter notation is written in Equation (5).

$$
\begin{aligned}
& \left\langle X, \operatorname{Ad}^{*}(x) \psi\right\rangle=\left\langle\operatorname{Ad}\left(x^{-1}\right) X, \psi\right\rangle \\
& \left(X \in \mathrm{g}, \psi \in \mathrm{g}^{*}, x \in G\right) .
\end{aligned}
$$

Another notion of a Lie group or a Lie algebra corresponding to Frobenius Lie algebra is a stabilizer of a Lie group or a Lie algebra. It can be considered a chosen linear functional in a dual space of $\mathfrak{g}$. Then, by using the coadjoint action of $\mathrm{Ad}^{*}$, the notion of stabilizer is obtained. Therefore, the stabilizer $G(\psi)$ of the Lie group of $G$ at a point $\psi \in \mathrm{g}^{*}$ is shown in Equation (6).

$$
G(\psi):=\left\{x \in G ; \quad \operatorname{Ad}^{*}(x) \psi=\psi\right\} \subset G .
$$

Furthermore, by taking the Lie algebra of $G(\psi)$, it can be considered the stabilizer of the Lie algebra at a point $\psi \in \mathfrak{g}^{*}$. This notion is equal to $\{0\}$ if $\mathfrak{g}$ is a Frobenius Lie algebra. This stabilizer of a Lie algebra can be written in the following formula.

$$
\mathrm{g}(\psi):=\left\{X \in g ; \operatorname{ad}^{*}(X) \psi=0\right\} \subset \mathrm{g}^{*} .
$$

In this case, the notion ad* is an action of $\mathrm{g}$ on its dual vector space $\mathrm{g}^{*}$. In other words, ad* is a derivation of the coadjoint action of $\mathrm{Ad}^{*}$. It can be written in the following formula.

$$
\operatorname{ad}^{*}(X): \mathrm{g}^{*} \ni \psi \mapsto \operatorname{ad}^{*}(X) \psi \in \mathrm{g}^{*} .
$$


Definition 4 (Fujiwara \& Ludwig, 2015). Let $\psi$ be an element of $\mathrm{g}^{*}$. A coadjoint orbit of $\Omega_{\psi}$ of a Lie group $\mathrm{G}$ is a set given in the following form.

$$
\operatorname{Ad}^{*}(G) \psi:=\left\{\operatorname{Ad}^{*}(x) \psi ; \quad x \in G\right\} \subset \mathrm{g}^{*} .
$$

Definition 5 (Csikós \& Verhóczki, 2007). A Lie algebra $\mathfrak{g}$ is said to be Frobenius if it has an open coadjoint orbit. In other words, a point of $\psi_{0}$ exists in the dual vector space $\mathrm{g}^{*}$, such that its stabilizer of $g\left(\psi_{0}\right)=\{0\}$.

To illustrate the explanation, the research shows an example from Fujiwara and Ludwig (2015). Let $\mathfrak{g}$ be a real Lie algebra spanned by a basis of $T=\left\{x_{1}, x_{2}\right\}$ . The non-zero brackets of $\mathfrak{g}$ are given by $\left[x_{1}, x_{2}\right]=x_{2}$. This Lie algebra $g$ can be written as a matrix realization in the following form.

$$
g \ni x=x^{1} x_{1}+x^{2} x_{2}=\left(\begin{array}{cc}
x^{1} & x^{2} \\
0 & 0
\end{array}\right) \text {. }
$$

Let $\mathrm{g}^{*}$ be a dual of the vector space of $\mathrm{g}$. Indeed, the elements $x_{1}^{*}$ and $x_{2}^{*}$ in $\mathrm{g}^{*}$ correspond to the elements $x_{1}$ and $x_{2}$ in $g$. In general, the element $f \in \mathrm{g}^{*}$ with respect to coordinates $T^{*}=\left\{x_{1}^{*}, x_{2}^{*}\right\}$ can be interpreted in the following matrix.

$$
g^{*} \ni f=\left(\begin{array}{ll}
x_{1}^{*} & * \\
x_{2}^{*} & *
\end{array}\right) .
$$

To relate the element $f \in \mathrm{g}^{*}$ with the element $x \in \mathrm{g}$, it is nice to apply a trace of a matrix. Using Equation (5), the elements $f \in \mathrm{g}^{*}$ and $x \in \mathrm{g}$ can be identified by the trace of the matrix multiplication. Then, it is obtained the following form.

$$
\langle x, f\rangle=\operatorname{tr}(f x)=x^{1} x_{1}^{*}+x^{2} x_{2}^{*} .
$$

Furthermore, the Lie group $\mathrm{G}$ of the Lie algebra $\mathfrak{g}$ has the matrix realization in Equation (13). It is easier to work in the matrix stage. To do that, let $e_{1}, e_{2}$ be elements of $\mathbb{R}$ with $e_{1}>0$, then the matrix realization for $\mathrm{G}$ is obtained as follows.

$$
G \in g=\left(\begin{array}{cc}
e_{1} & e_{2} \\
0 & 1
\end{array}\right) .
$$

By direct computations, there are two coadjoint orbits of $G$, which have the form in Equation (14). At the point of $\pm x_{2}^{*}$, the stabilizer of $g\left( \pm x_{2}^{*}\right)$ is trivial. In other words, the coadjoint orbits $\Omega_{ \pm x_{3}^{*}}$ in Equation (14) are open. Therefore, $\mathfrak{g}$ is the Frobenius Lie algebra of dimension 2 .

$$
\Omega_{ \pm x_{2}^{*}}=\left\{\left(x_{1}^{*}, x_{2}^{*}\right) ; \pm x_{2}^{*}>0\right\} \subset \mathrm{g}^{*} .
$$

Definition 6 (Gerstenhaber \& Giaquinto, 2009). Let $\mathfrak{g}$ be a Frobenius Lie algebra and $\mathfrak{g}^{*}$ be the dual vector space of $\mathfrak{g}$. The point $\psi_{0} \in \mathfrak{g}^{*}$ in satisfying $g\left(\psi_{0}\right)=\{0\}$ is called a Frobenius functional.
Let $\psi_{0} \in \mathrm{g}^{*}$ and $\mathrm{B}_{\psi_{0}}$ be an anti-symmetric bilinear form on $\mathfrak{g}$, it will become as follows.

$$
B_{\psi_{0}}: \mathrm{g} \times \mathrm{g} \ni(x, y) \mapsto\left\langle[x, y], \psi_{0}\right\rangle \in \mathbb{C} .
$$

The determinant of this anti-symmetric bilinear form in Equation (15) is equal to the determinant of the matrix $\mathrm{M}_{g}\left(\psi_{0}\right)$. The entries are given by Equation (16) with respect to a basis of $s=\left\{x_{1}, x_{2}, x_{3}, \ldots, x_{n}\right\}$ of $\mathfrak{g}$ with $n$ is the dimension of $\mathfrak{g}$. The Lie algebra of $\mathfrak{g}$ is a Frobenius if and only if the determinant $\operatorname{det}\left(\mathrm{M}_{g}\left(\psi_{0}\right)\right) \neq 0$ (Ooms, 1980). In other words, the Lie algebra of $\mathfrak{g}$ is a Frobenius if and only if the anti-symmetric bilinear form of $B_{\psi_{0}}$ is non-degenerate. This result is equivalent to the openness of the coadjoint orbit of $\Omega_{\psi_{0}}$ (Kurniadi \& Ishi, 2019).

$$
\mathrm{M}_{\mathrm{g}}\left(\psi_{0}\right)=\left\langle\left[x_{i}, y_{j}\right], \psi_{0}\right\rangle, \quad 1 \leq i, j \leq n .
$$

In the previous example, it can be seen that the Lie group $\mathrm{G}$ of the Lie algebra $\mathrm{g}=\operatorname{Span}\left\{x_{1}, x_{2}\right\}$ whose bracket is $\left[x_{1}, x_{2}\right]=x_{2}$ has two open coadjoint orbits as written in Equation (14). Thus, it can be found a Frobenius functional of $\mathfrak{g}$ of form $\psi_{0}= \pm x_{2}^{*}$. Furthermore, the matrix $\mathrm{M}_{\mathrm{g}}\left( \pm x_{2}^{*}\right)$ corresponding to the Frobenius functional can be considered as having a determinant of \pm 1 . It implies that $\mathfrak{g}$ is Frobenius Lie algebra.

$$
\mathrm{M}_{\mathfrak{g}}\left( \pm x_{2}^{*}\right)=\left(\begin{array}{cc}
0 & \pm 1 \\
\mp 1 & 0
\end{array}\right) .
$$

It is thought that to compute a Frobenius functional using coadjoint orbits computations is difficult. However, there is another alternative to find a Frobenius functional besides the computations of coadjoint orbits. It is introduced the notion of a Pfaffian of the square-alternating matrix $(X)$ of $2 k \times 2 k$ dimension. It has $\operatorname{det}(X)=\operatorname{Pf}(X)^{2}$. In this case, it constructs $\mathfrak{g}$-matrix with elements consisted of brackets in $\mathfrak{g}$. This matrix is named $\mathrm{g}\left(\left[x_{i}, x_{j}\right]\right]_{i, j=1}^{2 k}$. This Pfaffian is contained in a symmetric algebra of $\mathrm{S}(\mathfrak{g})$ of degree $k$. Then, the elements of $\mathfrak{g}^{*}$ correspond to the basis of the Lie algebra $\mathfrak{g}$. It forms the elements of symmetric algebra $\mathrm{S}(\mathrm{g})$, which are Frobenius functionals. This claim can be proven by Equation (16) if and only if $\operatorname{det}\left(\mathrm{M}_{\mathrm{g}}\left(\psi_{0}\right)\right) \neq 0$ (Ooms, 1980). Let $\mathrm{g}=\operatorname{Span}\left\{x_{1}, x_{2}\right\}$ be the Lie algebra whose bracket is given by $\left[x_{1}, x_{2}\right]=x_{2}$. It shows the formula as follows.

$$
\mathrm{g}\left(\left[x_{i}, x_{j}\right]\right)_{i, j=1}^{2}=\left(\begin{array}{cc}
0 & x_{2} \\
-x_{2} & 0
\end{array}\right) .
$$

It can be seen that the determinant $\mathrm{g}\left(\left[x_{i}, x_{j}\right]\right)_{i, j=1}^{2}$ is equal to $x_{2}^{2}$. Indeed, the Pfaffian $\operatorname{Pf}\left(g\left(\left[x_{i}, x_{j}\right]\right)_{i j=1}^{2}\right)=x_{2}$ is contained in symmetric algebra $\mathrm{s}\left(\mathrm{Span}\left\{x_{1}, x_{2}\right\}\right)$ of degree 1. It claims that the elements $\psi_{0}=x_{2}^{*}$ and $\psi_{0}=-x_{2}^{*}$ are Frobenius functionals. The claim is true based on Equation (17) since $\operatorname{det}\left(\mathrm{M}_{g}\left( \pm x_{2}^{*}\right)\right)= \pm 1$. The research shall apply this example to find Frobenius functionals 
in the proof of Proposition 1.

A Frobenius Lie algebra $\mathfrak{g}$ has even dimension (Ooms, 1980; Csikós \& Verhóczki, 2007). Indeed, at a Frobenius functional $\psi_{0} \in \mathrm{g}^{*}$, an anti-symmetric bilinear form $B_{\psi_{0}}$ defined on Equation (15) induces a symplectic form on a tangent space of the coadjoint orbit of $\Omega_{\psi_{0}}$. It is denoted by $T_{\psi_{0}} \Omega_{\psi_{0}}$. On the other hand, it is well known that the tangent space of $T_{\psi_{0}} \Omega_{\psi_{0}}$ is isomorphic to a quotient space $\mathrm{g} / \mathrm{g}\left(\psi_{0}\right)$. By definition of a Frobenius Lie algebra, $g\left(\psi_{0}\right)$ is trivial. Therefore, the dimension of a Frobenius Lie algebra is always even.

The notion of left-symmetric algebras plays an important role in the result. In the notion of mathematical physics, the left-symmetric algebra is called pre-Lie algebra (Burde, 2006). The leftsymmetric algebra comes in mathematical physics, particularly in renormalization theory. It is noted here that a Lie algebra equipped with a left-symmetric algebra can be embedded as a subalgebra of certain special linear Lie algebra of $\mathrm{sI}_{n}(\mathbb{R})$. It consists of $n \times n$ matrices of trace equalling to zero (Diatta \& Manga, 2014). Roughly speaking, a left-symmetric structure on a Lie algebra satisfying brackets of $\mathfrak{g}$ is called affine structures. The research will discuss this notion later. Let $\mathrm{A}$ be an algebra over $\mathrm{C}$ and $a, b, c$ be elements of $\mathrm{A}$ The associator of $(a, b, c)$ is in Equation (19). The * is a bilinear product on $\mathrm{A}$.

$$
\boldsymbol{J}\left(\alpha^{2} p^{\prime} c\right)=(\alpha * P) * C-\alpha *(p * C) \cdot
$$

Furthermore, left-symmetric structures on an algebra $\mathrm{A}$ are given by the following definition. Definition 7 (Burde, 2006). Let A be an algebra over C equipped with a bilinear product, as shown in Equation (20). Then, A is called a left-symmetric algebra if it satisfies Equation (21) for all a,b,c $\in \mathrm{A}$.

$$
\begin{aligned}
& \mathrm{A} \times \mathrm{A} \ni(a, b) \mapsto a b:=a * b \in \mathrm{A} . \\
& \beth(a, b, c)=\beth(b, a, c) .
\end{aligned}
$$

If Equation (21) is defined by Equation (22) for all a,b,c $\in \mathrm{A}$, the research uses the notion of rightsymmetric algebra. A left-symmetric algebra is the opposite of a right-symmetric algebra. It means that if $a * b$ is a product on a left-symmetric algebra, $a *_{R} b=b * a$ is a product on a right-symmetric algebra. If $A$ is a Lie algebra, such as $\mathfrak{g}$, the existence of affine structures can be shown.

$$
\beth(a, b, c)=\beth(a, c, b) .
$$

Definition 8 (Burde, 2006). Let $\mathfrak{g}$ be a Lie algebra. An affine structure on $\mathrm{g}$ is a bilinear product given by Equation (20) satisfying Equation (21) and a bracket in Equation (23) for all $\mathrm{a}, \mathrm{b} \in \mathrm{g}$.

$$
[a, b]=a b-b a \text {. }
$$

The two conditions in Equations (21) and (22) are equivalent to a Lie algebra homomorphism and a 1-cocycle. To do that, the notion of $\mathfrak{g}$-module, the space of 1-coboundaries, and the space of 1-cocycles are necessary to be recalled. Using Equation (21) and substituting Equation (23) to Equation (24), it shows several forms.

$$
\begin{aligned}
& (a b) c-(b a) c=a(b c)-b(a c) . \\
& {[a, b] c=a(b c)-b(a c) .}
\end{aligned}
$$

Firstly, Equation (25) is equivalent to the Lie algebra homomorphism. Let $L$ be a left multiplication on $\mathfrak{g}$ defined by $L(a) b=a b$ for all $a, b \in \mathfrak{g}$. As mentioned previously, Equation (25) is equivalent to a Lie algebra homomorphism $L$, and it shows the following form.

$$
\begin{aligned}
& L: \mathrm{g} \rightarrow \mathrm{gI}(\mathrm{g}) . \\
& {[L(a), L(b)] c=a(b c)-b(a c) .}
\end{aligned}
$$

Secondly, it shows that Equation (23) is equivalent to a 1-cocycle. Let $\mathfrak{g}_{L}$ be a $\mathfrak{g}$-module. In other words, Equation (23) is equivalent to a 1-cocyle of $\mathfrak{g}$-module $\mathfrak{g}_{L}$ in Equation (28) for $\psi=$ Id as an identity map on $\mathfrak{g}$.

$$
\begin{aligned}
& Z^{1}\left(\mathrm{~g}, \mathrm{~g}_{L}\right):=\left\{\psi \in \operatorname{Hom}\left(\mathrm{g}, \mathrm{g}_{L}\right)\right. \\
& \psi([a, b])=L(a) \psi(b)-L(b) \psi(a)\}
\end{aligned}
$$

Definition 9 (Diatta et al., 2020) Let $\mathfrak{g}$ be a Lie algebra and $\theta$ be any symplectic form on $\mathfrak{g}$. The symplectic structure $\mathfrak{g}$ induces a left-symmetric structure on $\mathfrak{g}$ defined by Equation (29).

$$
\theta(a b, c):=-\theta(b,[a, c]) \text {. }
$$

Definition 9 is very important in the research since it corresponds to the induction of a left-symmetric structure. On the other hand, each Frobenius Lie algebra has a Frobenius functional related to an antisymmetric bilinear form. In other words, by applying Equation (15), if $\mathfrak{g}$ is a Frobenius Lie algebra, it has the following form.

$$
\begin{aligned}
& B_{\psi}(a b, c)=-B_{\psi}(b,[a, c]) \\
& =-\langle[b,[a, c]], \psi\rangle .
\end{aligned}
$$

In this case, $B_{\psi}$ is the anti-symmetric bilinear form on the Frobenius Lie algebra of $\mathfrak{g}$ and $\psi$ is its Frobenius functional. Moreover, it can define a left multiplication on a Lie algebra $\mathfrak{g}$. It defines $R: g \rightarrow g I(g)$ using Equation (31) for every $a, b \in \mathrm{g}$.

$$
R(a) b=b a:=b * a .
$$

Definition 10 (Segal, 1992). Let $\mathfrak{g}$ be a Lie algebra equipped with a left-symmetric structure. The left-symmetric algebra $\mathrm{g}$ is called complete if a map in Equation (32) is bijective. 


$$
\mathrm{Id}+R(a): \mathrm{g} \rightarrow \mathrm{g} .
$$

Lemma 11 (Burde, 1998). Let $\mathfrak{g}$ be a leftsymmetric algebra. Then $\mathfrak{g}$ is complete if and only if it satisfies the following conditions. First, $g=\operatorname{rad}(g)$ with $\operatorname{rad}(\mathrm{g})$ is a radical of $\mathfrak{g}$. Second, the trace of $\mathrm{R}(\mathrm{a})$ equals 0 for all $a \in g$. Third, $R(a)$ has no eigenvalues in $\mathbb{C}-\{0\}$. Fourth, $\mathrm{R}(\mathrm{a})$ is a nilpotent linear map for all $\mathrm{a} \in \mathrm{g}$.

For example, a Lie algebra $\mathfrak{g}$ spanned by $S=\left\{x_{1}, x_{2}, x_{3}, x_{3}\right\}$ with non-zero brackets shown in Equation (33) has affine structures of the forms (Burde, 1998).

$$
\begin{aligned}
& {\left[x_{4}, x_{1}\right]=\left[x_{2}, x_{3}\right]=x_{1}} \\
& {\left[x_{2}, x_{4}\right]=x_{2}, \quad\left[x_{4}, x_{3}\right]=2 x_{3} .} \\
& x_{1} x_{2}=x_{2} x_{1}=x_{4}, \quad x_{3} x_{2}=x_{4} x_{1}=x_{1} \\
& x_{2} x_{3}=2 x_{1}, x_{4} x_{2}=-x_{2}, \quad x_{4} x_{3}=2 x_{3} .
\end{aligned}
$$

These structures are complete since for all $x \in \mathrm{g}$, the trace of $R(x)$ is equal to 0 . In contrast, in the previous example, the Lie algebra of $\mathfrak{g}$ spanned by basis $T=\left\{x_{1}, x_{2}\right\}$ with $\left[x_{1}, x_{2}\right]=x_{2}$ has affine structures in Equation (35) (Diatta \& Manga, 2014). However, the affine structure is not complete since there is $x_{1} \in \mathrm{g}$ with the trace of $R\left(x_{1}\right)=-2$, which is not equal to zero.

$$
\begin{aligned}
& x_{1}^{2}=-x_{1}, \quad x_{1} x_{2}=0, \\
& x_{2} x_{1}=-x_{2}, \quad x_{2}^{2}=0 .
\end{aligned}
$$

\section{METHODS}

The research applies an axiomatic method by studying some important notions corresponding to affine structures (Diatta et al., 2020), Borel subalgebras, and Frobenius Lie algebras (Csikós \& Verhóczki, 2007). It lets $\mathfrak{g}$ be the 6-dimensional Borel subalgebra of a simple Lie algebra. Since $\mathfrak{g}$ is isomorphic to the Frobenius Lie algebra, there exists a Frobenius functional of $\psi_{0}$. It implies that the alternating bilinear form of $B_{\psi_{0}}$ in Equation (15) is non-degenerate. Therefore, using alternating bilinear form $B_{\psi_{0}}$, the affine structure on 6-dimensional Borel subalgebra is induced from a Frobenius functional to prove the existence of affine structures. Moreover, the explicit formulas of the affine structures are also considered.

Figure 1 describes the process in the fishbone diagram. In the first step, the research can deduce that a given Lie algebra is the Borel subalgebra of a simple Lie algebra and the Frobenius Lie algebra (Csikós \& Verhóczki, 2007). However, the research gives detailed proof of its interest. In the second step, since the Borel subalgebra is Frobenius, a Frobenius functional of $\psi_{0}$ exists that the bilinear form $B_{\psi_{0}}$ defined by Equation (15) is non-degenerate or symplectic form. In the third and fourth steps, the research constructs a bilinear product in Equation (20).
Moreover, the symplectic form $B_{\psi_{0}}$ defined before inducing affine structures of the Borel subalgebra is in Equation (30). It proves the existence of affine structures on the Borel subalgebra. Finally, it obtains the formulas of affine structures written in Equation (2) and their completeness.

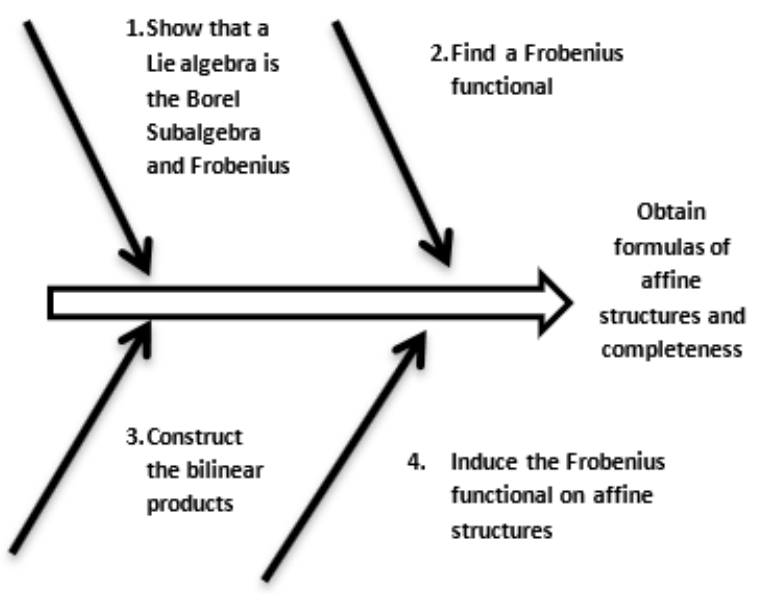

Figure 1 Fishbone Diagram

\section{RESULTS AND DISCUSSIONS}

In this section, the main results stated in Proposition 1 will be proven. The argument is divided into four parts as follows. First, the research will prove that the 6-dimensional Borel subalgebra of $g$ is Frobenius. Second, it will show the existence of affine structures on $\mathfrak{g}$. Third, it will give the explicit formulas of affine structures on $\mathfrak{g}$. Last, it will prove that affine structures are not complete.

Let $\mathfrak{g}$ be the given Lie algebra with basis $H=\left\{x_{i}\right\}_{i=1}^{6}$ that non-zero brackets are given by Equation (1). First, it shows that $g$ is the Borel subalgebra of a simple Lie algebra, and $\mathfrak{g}$ is the Frobenius Lie algebra. It defines that the Borel subalgebra is maximal solvable Lie subalgebra. To see it, the research considers the derived series on $\mathfrak{g}$ in Equation (36).

$$
\begin{aligned}
& g^{(0)}=g, \\
& g^{(1)}=\operatorname{Span}\left\{x_{3}, x_{4}, x_{5}, x_{6}\right\}, \\
& g^{(2)}=\operatorname{Span}\left\{x_{5}, x_{6}\right\}, \\
& g^{(3)}=\{0\} .
\end{aligned}
$$

Hence, there is a positive integer $k=3$, like $g^{(3)}=\{0\}$. The $\mathfrak{g}$ is solvable. Since this Lie algebra is a subalgebra of a simple Lie algebra, by using the definition of a simple Lie algebra, it shows that subalgebra $\mathfrak{g}$ is maximal. Thus, $\mathfrak{g}$ is the Borel subalgebra of a simple Lie algebra.

The research will complete the proof that $\mathfrak{g}$ is the Frobenius Lie algebra even though it is already shown by Csikós and Verhóczki (2007). It is denoted that $\mathrm{g}\left(\left[x_{i}, x_{j}\right]\right), 1 \leq i, j \leq 6$ is a matrix of $\mathrm{g}$-entries that the 
entries are defined by the brackets of $\mathfrak{g}$. Hence, it has Equation (37).

$\mathrm{g}\left(\left[x_{i}, x_{j}\right]\right)_{i, j=1}^{6}=\left(\begin{array}{cccccc}0 & 0 & x_{3} & 0 & x_{5} & 2 x_{6} \\ 0 & 0 & 0 & x_{4} & x_{5} & x_{6} \\ -x_{3} & 0 & 0 & x_{5} & x_{6} & 0 \\ 0 & -x_{4} & -x_{5} & 0 & 0 & 0 \\ -x_{5} & -x_{5} & -x_{6} & 0 & 0 & 0 \\ -2 x_{6} & -x_{6} & 0 & 0 & 0 & 0\end{array}\right)$.

This matrix is also called the structure matrix. The determinant of the structure matrix $g\left(\left[x_{i}, x_{j}\right]\right)_{i, j=1}^{6}$ can be considered. It implies the formula of Pfaffian $\operatorname{Pf}\left(g\left(\left[x_{i}, x_{j}\right]\right)_{i, j=1}^{6}\right)$, which is contained in a symmetric algebra $S(\mathfrak{g})$ of degree three. The formulas are obtained as follows.

$$
\begin{aligned}
& \operatorname{det}\left(g\left(\left[x_{i}, x_{j}\right]\right)_{i, j=1}^{6}\right)=x_{6}^{2}\left(x_{5}^{2}-2 x_{4} x_{6}\right)^{2} . \\
& \operatorname{Pf}\left(g\left(\left[x_{i}, x_{j}\right]\right)_{i, j=1}^{6}\right)=x_{6}\left(x_{5}^{2}-2 x_{4} x_{6}\right) .
\end{aligned}
$$

As discussed before, it is claimed that $\psi_{0}=x_{4}^{*}+x_{6}^{*} \in \mathrm{g}^{*}$ is the Frobenius functional. Of course, the research can choose another Frobenius functional in $\mathfrak{g}^{*}$ corresponding to Equation (39). To prove this, it obtains the matrix $\mathrm{M}_{\mathrm{g}}\left(\psi_{0}\right)$. The entries are defined in Equation (16). In other words, it just computes the value of the linear functional $\psi_{0}=x_{4}^{*}+x_{6}^{*}$ at the bracket of $\left[x_{i}, y_{j}\right]$. It shows $\left\langle\left[x_{i}, y_{j}\right], \psi_{0}\right\rangle, 1 \leq i, j \leq 6$, for every entry in the matrix $\mathrm{g}\left(\left[x_{i}, x_{j}\right]\right)_{i, j=1}^{6}$. Therefore, it results in Equation (40). Since it shows determinant $\mathrm{M}_{\mathrm{g}}\left(\psi_{0}\right)=4 \neq 0$, the claim is true. In other words, $\psi_{0}=x_{4}^{*}+x_{6}^{*} \in \mathrm{g}^{*}$ is the Frobenius functional. It implies that the Borel subalgebra is the Frobenius Lie algebra as desired.

$$
\mathrm{M}_{\mathrm{g}}\left(\psi_{0}\right)=\left(\begin{array}{cccccc}
0 & 0 & 0 & 0 & 0 & 2 \\
0 & 0 & 0 & 1 & 0 & 1 \\
0 & 0 & 0 & 0 & 1 & 0 \\
0 & -1 & 0 & 0 & 0 & 0 \\
0 & 0 & -1 & 0 & 0 & 0 \\
-2 & -1 & 0 & 0 & 0 & 0
\end{array}\right) .
$$

Second, it shows the existence of affine structures on the Borel subalgebra g. It defines the antisymmetric or alternating bilinear form of $B_{\psi_{0}}$ on $\mathfrak{g}$ in Equation (15). Indeed, $B_{\psi_{0}}$ is symplectic form because $\mathfrak{g}$ is the Frobenius Lie algebra. A bilinear product on $\mathfrak{g}$ is given Equation (20) for $x, y \in \mathfrak{g}, x y:=x * y \in \mathfrak{g}$. In addition, for any $x, y, z \in g$, it has Equation (41) and since elements $x, y, z \in g=\operatorname{Span}\left\{x_{1}, x_{2}, \ldots, x_{6}\right\}$, it can write them in Equation (42).

$$
\begin{aligned}
& B_{\psi_{0}}(x y, z)=-B_{\psi_{0}}(y,[x, z]) \\
& =-\left\langle[y,[x, z]], \psi_{0}\right\rangle \\
& =-\left\langle[y,[x, z]], x_{4}^{*}+x_{6}^{*}\right) .
\end{aligned}
$$

$$
\begin{aligned}
& x=\alpha_{1} x_{1}+\alpha_{2} x_{2}+\cdots+\alpha_{6} x_{6}, \\
& y=\beta_{1} x_{1}+\beta_{2} x_{2}+\cdots+\beta_{6} x_{6}, \\
& z=\gamma_{1} x_{1}+\gamma_{2} x_{2}+\cdots+\gamma_{6} x_{6} .
\end{aligned}
$$

In this term, let $z$ be the product of $x$ and $y$. If it computes Equation (41) with respect to the basis $H=\left\{x_{i}\right\}_{i=1}^{6}$, it will obtain the scalars of $\gamma_{k}, k=1,2, \ldots, 6$ written in the terms $\alpha_{i}$ and $\beta_{i}, 1 \leq i, j \leq 6$. In other words, $B_{\psi_{0}}\left(x y, x_{k}\right), k=1,2, \ldots, 6$ is not all zero. It happens because the stabilizer of $\mathrm{g}\left(\psi_{0}\right)$ is trivial, such as $\mathrm{g}\left(\psi_{0}\right)=\{0\}$. Thus, there are affine structures on the Borel subalgebra of $\mathfrak{g}$.

Third, the research gives the detailed formulas of affine structures on $\mathrm{g}$ as follows. It computes for $z=x_{1}$ completely, and another follows these computations in a similar way. Moreover, Equation (43) can be considered, and using Equations (1) and (42), Equation (44) is also obtained.

$$
\begin{aligned}
& B_{\psi_{0}}\left(x y, x_{1}\right)=-B_{\psi_{0}}\left(y,\left[x, x_{1}\right]\right) \\
& =-\left\langle\left[y,\left[x, x_{1}\right]\right], x_{4}^{*}+x_{6}^{*}\right\rangle . \\
& {\left[x, x_{1}\right]=\left[\alpha_{1} x_{1}+\alpha_{2} x_{2}+\cdots+\alpha_{6} x_{6}, x_{1}\right]} \\
& =-\alpha_{3} x_{3}-\alpha_{5} x_{5}-2 \alpha_{6} x_{6} .
\end{aligned}
$$

Equations (45) and (46) are computed by substituting Equation (44) to $\left[y,\left[x, x_{1}\right]\right]$ and substituting Equations (43) to (45). These computations are based on the Borel subalgebra brackets in Equation (1). Moreover, the induced left-symmetric algebra helps to get these formulas. On the other hand, Equations (47) and (48) are obtained by direct calculations of antisymmetric bilinear form $B_{\psi_{0}}\left(x y, x_{1}\right)$.

$$
\begin{aligned}
& {\left[y,-\alpha_{3} x_{3}-\alpha_{5} x_{5}-2 \alpha_{6} x_{6}\right]} \\
& =\left[\beta_{1} x_{1}+\beta_{2} x_{2}+\cdots+\beta_{6} x_{6},-\alpha_{3} x_{3}-\alpha_{5} x_{5}-2 \alpha_{6} x_{6}\right] \\
& =\left[\beta_{1} x_{1}+\beta_{2} x_{2}+\cdots+\beta_{6} x_{6},-\alpha_{3} x_{3}\right] \\
& +\left[\beta_{1} x_{1}+\beta_{2} x_{2}+\cdots+\beta_{6} x_{6},-\alpha_{5} x_{5}\right] \\
& +\left[\beta_{1} x_{1}+\beta_{2} x_{2}+\cdots+\beta_{6} x_{6},-2 \alpha_{6} x_{6}\right] \\
& =\beta_{1} \alpha_{3} x_{3}-\beta_{4} \alpha_{3} x_{5}-\beta_{5} \alpha_{3} x_{6}+\beta_{1} \alpha_{5} x_{5} \\
& +\beta_{2} \alpha_{5} x_{5}+\beta_{3} \alpha_{5} x_{6}+4 \beta_{1} \alpha_{6} x_{6}+2 \beta_{2} \alpha_{6} x_{6} . \\
& B_{\psi_{0}}\left(x y, x_{1}\right)=-\beta_{5} \alpha_{3}+\beta_{3} \alpha_{5}+4 \beta_{1} \alpha_{6}+2 \beta_{2} \alpha_{6} . \\
& B_{\psi_{0}}\left(\gamma_{1} x_{1}+\gamma_{2} x_{2}+\cdots+\gamma_{6} x_{6}, x_{1}\right)=-2 \gamma_{6} . \\
& \gamma_{6}=\frac{1}{2}\left(\beta_{5} \alpha_{3}-\beta_{3} \alpha_{5}-4 \beta_{1} \alpha_{6}-2 \beta_{2} \alpha_{6}\right) .
\end{aligned}
$$

The left computations are $\gamma_{1}, \gamma_{2}, \gamma_{3}, \gamma_{4}$, and $\gamma_{5}$. The computations follow $\gamma_{6}$ with respect to the Borel subalgebra brackets. In other words, the formulas are written in Equation (49).

$$
\begin{aligned}
& \gamma_{4}=-\left(\beta_{2} \alpha_{4}+\beta_{5} \alpha_{5}\right)-\frac{1}{2}\left(\beta_{5} \alpha_{3}-\beta_{3} \alpha_{5}\right), \\
& \gamma_{5}=-\left(\beta_{5} \alpha_{1}+\beta_{3} \alpha_{4}+2 \beta_{1} \alpha_{5}+\beta_{2} \alpha_{5}\right), \\
& \gamma_{2}=-\left(\beta_{2} \alpha_{2}+\beta_{3} \alpha_{3}\right),
\end{aligned}
$$




$$
\begin{aligned}
& \gamma_{3}=-\left(\beta_{3} \alpha_{1}+\beta_{3} \alpha_{2}+2 \beta_{1} \alpha_{3}+\beta_{2} \alpha_{3}\right), \\
& \gamma_{1}=-2 \beta_{1} \alpha_{1}-\beta_{2} \alpha_{1}-\beta_{1} \alpha_{2}+\frac{1}{2} \beta_{3} \alpha_{3} .
\end{aligned}
$$

It obtains the products $x y$ in Equation (50) with $\gamma_{i}, 1 \leq i, j \leq 6$ It is considered by Equations (48) and (49). Choosing the suitable $\alpha_{i}$ and $\beta, 1 \leq i, j \leq 6$, it has the desired formula in Equation (2), which is nothing but affine structures on the 6-dimensional Borel subalgebra of $\mathfrak{g}$.

$$
x y=\gamma_{1} x_{1}+\gamma_{2} x_{2}+\cdots+\gamma_{6} x_{6} .
$$

Last, for affine structures, the research chooses the point $x_{1} \in H \subset \mathrm{g}$. The matrix representation of $R\left(x_{1}\right)$ is shown by Equation (51) that the trace is equal to -8 . Using Lemma 10, affine structures on $\mathrm{g}$ are not complete.

$$
R=\left(\begin{array}{cccccc}
-2 & -1 & 0 & 0 & 0 & 2 \\
0 & 0 & 0 & 0 & 0 & 0 \\
0 & 0 & -2 & 0 & 1 & 0 \\
0 & 0 & 0 & 0 & 0 & 0 \\
0 & 0 & 0 & 0 & -2 & 0 \\
0 & 0 & 0 & 0 & 0 & -2
\end{array}\right) .
$$

The main result equips the results in Burde (2006) about Milnor's question of the existence of complete affine structures on a solvable Lie algebra. The results show that not every solvable Lie algebra has complete affine structures. The affine structures and their completeness on remain isomorphism classes of 6-dimensional Frobenius Lie algebras still need to be studied.

In the lower dimension case, there are three isomorphism classes of 4-dimensional Frobenius Lie algebra over a field of characteristic $\neq 2$ (Csikós \& Verhóczki, 2007). The research conjectures that all 4-dimensional real Frobenius Lie algebras are solvable. Another previous research computes the coadjoint orbits of Lie groups of 4-dimensional real Frobenius Lie algebras (Kurniadi \& Ishi, 2019). Therefore, it can find all Frobenius functionals. The research also conjectures that these Frobenius Lie algebras have affine structures which are not complete. It needs to investigate more about these statements for future research.

\section{CONCLUSIONS}

The notion of affine structures arises in convex homogeneous cones, vertex algebras, and affine manifolds. On the other hand, it is well known that Frobenius Lie algebras correspond to the research of bounded homogeneous domains in $\mathbb{C}^{n}$. Furthermore, one of 16 isomorphism classes of 6-dimensional Frobenius Lie algebras over an algebraically closed field is the Borel subalgebra. The research studies the affine structures for the 6-dimensional Borel subalgebra of a simple Lie algebra. The result shows that the 6-dimensional Borel subalgebra of a simple Lie algebra has affine structures. The research also gives the explicit formulas of affine structures on this Lie algebra in Equation (2). It proves that the affine structure on this solvable Lie algebra is not complete. The main research is stated and proven in Proposition 1 .

In the research, the result is just for the 6-dimensional Borel subalgebra. Therefore, for future research, there are 15 isomorphism classes of 6-dimensional Frobenius Lie algebra that still need to be investigated whether it has affine structures or not. Furthermore, it is interesting to consider whether these affine structures complete or not.

\section{ACKNOWLEDGEMENTS}

The first author is very grateful to Professor Hideyuki Ishi from Osaka City University, Japan, for the generous support and guidance to the first author to study Frobenius Lie algebras. The authors would like to thank reviewers for their worthy advice on the research. The research was supported by a grant from Universitas Padjadjaran through Riset Percepatan Lektor Kepala (RPLK) in 2020 with Contract Number: 1427/UN6.3.1/LT/2020. The authors would like to thank Universitas Padjadjaran which has funded the research.

\section{REFERENCES}

Alvarez, M. A., Rodríguez-Vallarte, M. C., \& Salgado, G. (2018). Contact and Frobenius solvable Lie algebras with Abelian Nilradical. Communications in Algebra, 46(10), 4344-4354. https://doi.org/10.1 080/00927872.2018.1439048

Burde, D. (2006). Left-symmetric algebras, or pre-Lie algebras in geometry and physics. Central European Journal of Mathematics, 4(3), 323-357.

Burde, D. (1998). Simple left-symmetric algebras with 9 solvable Lie algebra. Manuscripta Mathematica, 95(3), 397-411.

Csikós, B., \& Verhóczki, L. (2007). Classification of Frobenius Lie algebras of dimension $\leq 6$. Publicationes Mathematicae-Debrecen, 70(3-4), 427-451.

Diatta, A., Manga, B., \& Mbaye, A. (2020). On systems of commuting matrices, Frobenius Lie algebras and Gerstenhaber's Theorem. ArXiv:2002.08737.

Diatta, A., \& Manga, B. (2014). On properties of principal elements of Frobenius Lie algebras. Journal of Lie Theory, 24(3), 849-864.

Fujiwara, H., \& Ludwig, J. (2015). Harmonic analysis on exponential solvable Lie groups. Springer.

Gerstenhaber, M., \& Giaquinto, A. (2009). The principal element of a Frobenius Lie algebra. Letters in Mathematical Physics, 88, 333-341. https://doi. org/10.1007/s11005-009-0321-8

Hilgert, J., \& Neeb, K. H. (2011). Structure and geometry of Lie groups. Springer Science \& Business Media.

Kurniadi, E. (2019). Harmonic analysis for finite dimensional real Frobenius Lie algebras (Doctoral 
dissertation). Nagoya University.

Kurniadi, E., \& Ishi, H. (2019). Harmonic analysis for 4-dimensional real Frobenius Lie algebras. In International Conference on Geometric and Harmonic Analysis on Homogeneous Spaces and Applications (pp. 95-109).

Ooms, A. I. (1980). On Frobenius Lie algebras. Communications in Algebra, 8, 13-52. https://doi. org/10.1080/00927878008822445

Segal, D. (1992). The structure of complete left-symmetric algebras. Mathematische Annalen, 293(1), 569-578. 